\title{
Monotonicity properties for a ratio of finite many gamma functions
}

Feng Qi $i^{1,2,3}$ (D) and Dongkyu Lim ${ }^{4^{*}}$ (D)

Dedicated to people facing and fighting COVID-19

${ }^{*}$ Correspondence: dklim@anu.ac.kr

${ }^{4}$ Department of Mathematics

Education, Andong National

University, Andong, Republic of

Korea

Full list of author information is

available at the end of the article

\begin{abstract}
In the paper, the authors consider a ratio of finite many gamma functions and find its monotonicity properties such as complete monotonicity, the Bernstein function property, and logarithmically complete monotonicity.
\end{abstract}

\section{Preliminaries}

Let $f(x)$ be an infinite differentiable function on an infinite interval $(0, \infty)$.

(1) If $(-1)^{k} f^{(k)}(x) \geq 0$ for all $k \geq 0$ and $x \in(0, \infty)$, then we call $f(x)$ a completely monotonic function on $(0, \infty)$. See the review papers $[22,31,36]$ and $[35$, Chapter IV].

(2) If $(-1)^{k}[\ln f(x)]^{(k)} \geq 0$ for all $k \geq 1$ and $x \in(0, \infty)$, or say, if the logarithmic derivative $[\ln f(x)]^{\prime}=\frac{f^{\prime}(x)}{f(x)}$ is a completely monotonic function on $(0, \infty)$, then we call $f(x)$ a logarithmically completely monotonic function on $(0, \infty)$. See the papers $[3,4,7,24]$ and [33, Chap. 5].

(3) If $f^{\prime}(x)$ is a completely monotonic function on $(0, \infty)$, then we call $f(x)$ a Bernstein function on $(0, \infty)$. See the paper [28] and the monograph [33].

The classical gamma function $\Gamma(z)$ can be defined by

$$
\Gamma(z)=\int_{0}^{\infty} t^{z-1} e^{-t} \mathrm{~d} t, \quad \Re(z)>0
$$

or by

$$
\Gamma(z)=\lim _{n \rightarrow \infty} \frac{n ! n^{z}}{\prod_{k=0}^{n}(z+k)}, \quad z \in \mathbb{C} \backslash\{0,-1,-2, \ldots\}
$$

See [1, Chap. 6], [15, Chap. 5], the paper [18], and [34, Chap. 3]. In the literature, the logarithmic derivative

$$
\psi(z)=[\ln \Gamma(x)]^{\prime}=\frac{\Gamma^{\prime}(z)}{\Gamma(z)}
$$

(c) The Author(s) 2020. This article is licensed under a Creative Commons Attribution 4.0 International License, which permits use, sharing, adaptation, distribution and reproduction in any medium or format, as long as you give appropriate credit to the original author(s) and the source, provide a link to the Creative Commons licence, and indicate if changes were made. The images or other third party material in this article are included in the article's Creative Commons licence, unless indicated otherwise in a credit line to the material. If material is not included in the article's Creative Commons licence and your intended use is not permitted by statutory regulation or exceeds the permitted use, you will need to obtain permission directly from the copyright holder. To view a copy of this licence, visit http://creativecommons.org/licenses/by/4.0/. 
and its first derivative $\psi^{\prime}(z)$ are respectively called the digamma and trigamma functions. See the papers $[5,6,10,25,26]$ and closely related references therein.

\section{Motivations}

This paper is motivated by a series of papers [2, 11, 12, 16, 19, 21, 27, 29, 32]. For detailed review and survey, please read the papers $[19,27,29,32]$ and closely related references therein.

In the paper [2], motivated by $[11,12]$, the function

$$
x \in(0, \infty) \mapsto \frac{\Gamma(n x+1)}{\Gamma(k x+1) \Gamma((m-k) x+1)} p^{k x}(1-p)^{(m-k) x}
$$

was considered, where $p \in(0,1)$ and $k, m$ are nonnegative integers with $0 \leq k \leq m$.

In [16, Theorem 2.1] and [32], the function

$$
x \in(0, \infty) \mapsto \frac{\Gamma\left(1+x \sum_{i=1}^{m} \lambda_{i}\right)}{\prod_{i=1}^{m} \Gamma\left(1+x \lambda_{i}\right)} \prod_{i=1}^{m} p_{i}^{x \lambda_{i}}
$$

was independently studied, where $m \geq 2, \lambda_{i}>0$ for $1 \leq i \leq m, p_{i} \in(0,1)$ for $1 \leq i \leq m$, and $\sum_{i=1}^{m} p_{i}=1$. The $q$-analogue

$$
x \in(0, \infty) \mapsto \frac{\Gamma_{q}\left(1+x \sum_{i=1}^{m} \lambda_{i}\right)}{\prod_{i=1}^{m} \Gamma_{q}\left(1+x \lambda_{i}\right)} \prod_{i=1}^{m} p_{i}^{x \lambda_{i}}
$$

of the function in (2.2) was investigated in [19], where $q \in(0,1), m \geq 2, \lambda_{i}>0$ for $1 \leq$ $i \leq m, p_{i} \in(0,1)$ for $1 \leq i \leq m$ with $\sum_{i=1}^{m} p_{i}=1$, and $\Gamma_{q}$ is the $q$-analogue of the gamma function $\Gamma$.

The functions

$$
x \in(0, \infty) \mapsto \frac{\prod_{i=1}^{m} \Gamma\left(v_{i} x+1\right) \prod_{j=1}^{n} \Gamma\left(\tau_{j} x+1\right)}{\prod_{i=1}^{m} \prod_{j=1}^{n} \Gamma\left(\lambda_{i j} x+1\right)}
$$

and

$$
x \in(0, \infty) \mapsto \frac{\prod_{i=1}^{m} \Gamma\left(1+v_{i} x\right) \prod_{j=1}^{n} \Gamma\left(1+\tau_{j} x\right)}{\left[\prod_{i=1}^{m} \prod_{j=1}^{n} \Gamma\left(1+\lambda_{i j} x\right)\right]^{\rho}}
$$

were respectively considered in [17, Theorem 2.1] and [29, Theorem 4.1], where $\rho \in \mathbb{R}$ and $\lambda_{i j}>0, v_{i}=\sum_{j=1}^{n} \lambda_{i j}, \tau_{j}=\sum_{i=1}^{m} \lambda_{i j}$ for $1 \leq i \leq m$ and $1 \leq j \leq n$.

In [27], the function

$$
x \in(0, \infty) \mapsto \frac{\prod_{i=1}^{m}\left[\Gamma\left(1+v_{i} x\right)\right]^{v_{i}^{\theta}} \prod_{j=1}^{n}\left[\Gamma\left(1+\tau_{j} x\right)\right]^{\tau_{j}^{\theta}}}{\prod_{i=1}^{m} \prod_{j=1}^{n}\left[\Gamma\left(1+\lambda_{i j} x\right)\right]^{\rho \lambda_{i j}^{\theta}}}
$$

was discussed, where $\rho, \theta \in \mathbb{R}$ and $\lambda_{i j}>0, v_{i}=\sum_{j=1}^{n} \lambda_{i j}, \tau_{j}=\sum_{i=1}^{m} \lambda_{i j}$ for $1 \leq i \leq m$ and $1 \leq j \leq n$. 
In this paper, stimulated by the above six functions (2.1), (2.2), (2.3), (2.4), (2.5), and (2.6), we consider a new function

$$
\mathcal{Q}(x)=\mathcal{Q}_{m, a, p, \rho, \varrho, \theta}(x)=\frac{\left[\Gamma\left(1+x \sum_{i=1}^{m} a_{i}\right)\right]^{\left(\sum_{i=1}^{m} a_{i}\right)^{\theta}}}{\prod_{i=1}^{m}\left[\Gamma\left(1+x a_{i}\right)\right]^{\rho a_{i}^{\theta}}}\left(\prod_{i=1}^{m} p_{i}^{a_{i}}\right)^{\varrho x}
$$

on $(0, \infty)$, where $m \geq 2, \rho, \varrho, \theta \in \mathbb{R}, a=\left(a_{1}, a_{2}, \ldots, a_{m}\right)$ with $a_{i}>0$ for $1 \leq i \leq m$, and $p=$ $\left(p_{1}, p_{2}, \ldots, p_{m}\right)$ with $p_{i} \in(0,1)$ for $1 \leq i \leq m$ and $\sum_{i=1}^{m} p_{i}=1$.

\section{Monotonicity properties}

In this section, we now start out to find and prove some monotonicity properties for the function $\mathcal{Q}(x)=\mathcal{Q}_{m, a, p, \rho, e, \theta}(x)$ defined in (2.7). Our main results in this section can be stated in the following theorem.

Theorem 3.1 Let $m \geq 2, a=\left(a_{1}, a_{2}, \ldots, a_{m}\right)$ with $a_{i}>0$ for $1 \leq i \leq m$, and $p=\left(p_{1}, p_{2}, \ldots\right.$, $\left.p_{m}\right)$ with $\sum_{i=1}^{m} p_{i}=1$ and $p_{i} \in(0,1)$ for $1 \leq i \leq m$. Then

(1) when $\rho \leq 1$ and $\theta \geq 0$, the second logarithmic derivative

$$
[\ln \mathcal{Q}(x)]^{\prime \prime}=\left(\sum_{i=1}^{m} a_{i}\right)^{\theta+2} \psi^{\prime}\left(1+x \sum_{i=1}^{m} a_{i}\right)-\rho \sum_{i=1}^{m} a_{i}^{\theta+2} \psi^{\prime}\left(1+a_{i} x\right)
$$

is completely monotonic on $(0, \infty)$;

(2) when $\rho=1, \varrho=0$, and $\theta=0$, the function

$$
\mathcal{Q}_{m, a, p, 1,0,0}(x)=\frac{\Gamma\left(1+x \sum_{i=1}^{m} a_{i}\right)}{\prod_{i=1}^{m} \Gamma\left(1+x a_{i}\right)}
$$

is increasing on $(0, \infty)$ and its logarithmic derivative

$$
\left[\ln \mathcal{Q}_{m, a, p, 1,0,0}(x)\right]^{\prime}=\left(\sum_{i=1}^{m} a_{i}\right) \psi\left(1+x \sum_{i=1}^{m} a_{i}\right)-\sum_{i=1}^{m} a_{i} \psi\left(1+a_{i} x\right)
$$

is a Bernstein function on $(0, \infty)$;

(3) when $\rho=1, \varrho \geq 1$, and $\theta=0$, the function $\mathcal{Q}_{m, a, p, 1, e, 0}(x)$ is logarithmically completely monotonic on $(0, \infty)$;

(4) when $(\rho, \varrho, \theta) \in S$ and

$$
S=\{\rho \leq 1, \varrho \geq 0, \theta \geq 0\} \backslash\{\rho=1, \varrho=0, \theta=0\} \backslash\{\rho=1, \varrho \geq 1, \theta=0\},
$$

the function $\mathcal{Q}_{m, a, p, \rho, e, \theta}(x)$ has a unique minimum on $(0, \infty)$.

Proof Direct calculation gives

$$
\begin{aligned}
& \ln \mathcal{Q}(x)=\left(\sum_{i=1}^{m} a_{i}\right)^{\theta} \ln \Gamma\left(1+x \sum_{i=1}^{m} a_{i}\right)-\rho \sum_{i=1}^{m} a_{i}^{\theta} \ln \Gamma\left(1+a_{i} x\right)+\varrho x \sum_{i=1}^{m} a_{i} \ln p_{i}, \\
& {[\ln \mathcal{Q}(x)]^{\prime}=\left(\sum_{i=1}^{m} a_{i}\right)^{\theta+1} \psi\left(1+x \sum_{i=1}^{m} a_{i}\right)-\rho \sum_{i=1}^{m} a_{i}^{\theta+1} \psi\left(1+a_{i} x\right)+\varrho \sum_{i=1}^{m} a_{i} \ln p_{i},}
\end{aligned}
$$


and

$$
[\ln \mathcal{Q}(x)]^{\prime \prime}=\left(\sum_{i=1}^{m} a_{i}\right)^{\theta+2} \psi^{\prime}\left(1+x \sum_{i=1}^{m} a_{i}\right)-\rho \sum_{i=1}^{m} a_{i}^{\theta+2} \psi^{\prime}\left(1+a_{i} x\right)
$$

As in $[27,29,32]$, from

$$
\psi^{\prime}(z)=\int_{0}^{\infty} \frac{t}{1-e^{-t}} e^{-z t} \mathrm{~d} t, \quad \Re(z)>0
$$

in $[1, \mathrm{p} .260,6.4 .1]$, it follows that

$$
\psi^{\prime}(1+\tau z)=\int_{0}^{\infty} \frac{t}{1-e^{-t}} e^{-(1+\tau z) t} \mathrm{~d} t=\frac{1}{\tau} \int_{0}^{\infty} h\left(\frac{v}{\tau}\right) e^{-v z} \mathrm{~d} \nu
$$

where $\tau>0$ and $h(t)=\frac{t}{e^{t}-1}$ is the generating function of the classical Bernoulli numbers, see $[20,23]$ and [34, Chap. 1]. Accordingly, we have

$$
[\ln \mathcal{Q}(x)]^{\prime \prime}=\int_{0}^{\infty}\left[\left(\sum_{i=1}^{m} a_{i}\right)^{\theta+1} h\left(\frac{v}{\sum_{i=1}^{m} a_{i}}\right)-\rho \sum_{i=1}^{m} a_{i}^{\theta+1} h\left(\frac{v}{a_{i}}\right)\right] e^{-v x} \mathrm{~d} v
$$

In [27, Theorem 4.1], it was discovered that

$$
\sum_{i=1}^{m} \frac{v_{i}^{\alpha}}{e^{x / v_{i}}-1}+\sum_{j=1}^{n} \frac{\tau_{j}^{\alpha}}{e^{x / \tau_{j}}-1} \geq 2 \sum_{i=1}^{m} \sum_{j=1}^{n} \frac{\lambda_{i j}^{\alpha}}{e^{x / \lambda_{i j}}-1}
$$

where $\alpha \geq 0, x>0, \lambda_{i j}>0$ for $1 \leq i \leq m$ and $1 \leq j \leq n, v_{i}=\sum_{j=1}^{n} \lambda_{i j}$, and $\tau_{j}=\sum_{i=1}^{m} \lambda_{i j}$. As remarked in [27, Remark 4.1], setting $n=m$ and $\lambda_{1 k}=\lambda_{k 1}=\lambda_{k}>0$ for $1 \leq k \leq m$ and letting $\lambda_{i j} \rightarrow 0^{+}$for $2 \leq i, j \leq m$ in inequality (3.2) result in

$$
\frac{\left(\sum_{k=1}^{m} \lambda_{k}\right)^{\alpha}}{e^{x / \sum_{k=1}^{m} \lambda_{k}}-1} \geq \sum_{k=1}^{m} \frac{\lambda_{k}^{\alpha}}{e^{x / \lambda_{k}}-1}
$$

for $x>0, \lambda_{k}>0$, and $\alpha \geq 0$. Inequality (3.3) can be equivalently formulated as

$$
\left(\sum_{k=1}^{m} \lambda_{k}\right)^{\alpha+1} h\left(\frac{x}{\sum_{k=1}^{m} \lambda_{k}}\right) \geq \sum_{k=1}^{m} \lambda_{k}^{\alpha+1} h\left(\frac{x}{\lambda_{k}}\right)
$$

for $x>0, \lambda_{k}>0$, and $\alpha \geq 0$.

Combining inequality (3.4) with equation (3.1) yields that, when $\rho \leq 1$ and $\theta \geq 0$, the second derivative $[\ln \mathcal{Q}(x)]^{\prime \prime}$ is completely monotonic on $(0, \infty)$.

The complete monotonicity of $[\ln \mathcal{Q}(x)]^{\prime \prime}$ implies that the first derivative $[\ln \mathcal{Q}(x)]^{\prime}$ is strictly increasing on $(0, \infty)$. Therefore, by virtue of the limit

$$
\lim _{x \rightarrow \infty}[\ln x-\psi(x)]=0
$$


in [8, Theorem 1] and [9, Sect. 1.4], we have

$$
\begin{aligned}
\lim _{x \rightarrow 0^{+}}[\ln \mathcal{Q}(x)]^{\prime}= & \lim _{x \rightarrow 0^{+}}\left[\left(\sum_{i=1}^{m} a_{i}\right)^{\theta+1} \psi\left(1+x \sum_{i=1}^{m} a_{i}\right)-\rho \sum_{i=1}^{m} a_{i}^{\theta+1} \psi\left(1+a_{i} x\right)\right] \\
& +\varrho \sum_{i=1}^{m} a_{i} \ln p_{i} \\
= & \psi(1)\left[\left(\sum_{i=1}^{m} a_{i}\right)^{\theta+1}-\rho \sum_{i=1}^{m} a_{i}^{\theta+1}\right]+\varrho \sum_{i=1}^{m} a_{i} \ln p_{i} \\
& \left\{\begin{array}{l}
=0, \quad \theta=0, \rho=1, \varrho=0 ; \\
<0, \quad \theta=0, \rho=1, \varrho>0 ; \\
<0, \quad \theta=0, \rho<1, \varrho \geq 0 \\
<0, \quad \theta>0, \rho \leq 1, \varrho \geq 0
\end{array}\right.
\end{aligned}
$$

where $\psi(1)=-0.577 \ldots$, and

$$
\begin{aligned}
& \lim _{x \rightarrow \infty}[\ln \mathcal{Q}(x)]^{\prime}=\lim _{x \rightarrow \infty}\left[\left(\sum_{i=1}^{m} a_{i}\right)^{\theta+1} \psi\left(1+x \sum_{i=1}^{m} a_{i}\right)-\rho \sum_{i=1}^{m} a_{i}^{\theta+1} \psi\left(1+a_{i} x\right)\right] \\
& +\varrho \sum_{i=1}^{m} a_{i} \ln p_{i} \\
& =\lim _{x \rightarrow \infty}\left\{\left(\sum_{i=1}^{m} a_{i}\right)^{\theta+1}\left[\psi\left(1+x \sum_{i=1}^{m} a_{i}\right)-\ln \left(1+x \sum_{i=1}^{m} a_{i}\right)\right]\right. \\
& \left.-\rho \sum_{i=1}^{m} a_{i}^{\theta+1}\left[\psi\left(1+a_{i} x\right)-\ln \left(1+a_{i} x\right)\right]\right\}+\varrho \sum_{i=1}^{m} a_{i} \ln p_{i} \\
& +\lim _{x \rightarrow \infty}\left[\left(\sum_{i=1}^{m} a_{i}\right)^{\theta+1} \ln \left(1+x \sum_{i=1}^{m} a_{i}\right)-\rho \sum_{i=1}^{m} a_{i}^{\theta+1} \ln \left(1+a_{i} x\right)\right] \\
& =\varrho \sum_{i=1}^{m} a_{i} \ln p_{i}+\lim _{x \rightarrow \infty} \ln \frac{\left(1+x \sum_{i=1}^{m} a_{i}\right)^{\left(\sum_{i=1}^{m} a_{i}\right)^{\theta+1}}}{\prod_{i=1}^{m}\left(1+a_{i} x\right)^{\rho a_{i}^{\theta+1}}} \\
& =\ln \lim _{x \rightarrow \infty} \frac{\left(\frac{1}{x}+\sum_{i=1}^{m} a_{i}\right)^{\left(\sum_{i=1}^{m} a_{i}\right)^{\theta+1}}}{\prod_{i=1}^{m}\left(\frac{1}{x}+a_{i}\right)^{\rho a_{i}^{\theta+1}}} \\
& +\ln \lim _{x \rightarrow \infty} x^{\left(\sum_{i=1}^{m} a_{i}\right)^{\theta+1}-\rho \sum_{i=1}^{m} a_{i}^{\theta+1}}+\varrho \sum_{i=1}^{m} a_{i} \ln p_{i} \\
& =\varrho \sum_{i=1}^{m} a_{i} \ln p_{i}+\ln \frac{\left(\sum_{i=1}^{m} a_{i}\right)^{\left(\sum_{i=1}^{m} a_{i}\right)^{\theta+1}}}{\left(\prod_{i=1}^{m} a_{i}^{a_{i}^{\theta+1}}\right)^{\rho}} \\
& + \begin{cases}0, & \rho=\frac{\left(\sum_{i=1}^{m} a_{i}\right)^{\theta+1}}{\sum_{i=1}^{m} a_{i}^{\theta+1}} ; \\
-\infty, & \rho>\frac{\left(\sum_{i=1}^{m} a_{i}\right)^{\theta+1}}{\sum_{i=1}^{m} a_{i}^{\theta+1}} \\
\infty, & \rho<\frac{\left(\sum_{i=1}^{m} a_{i}\right)^{\theta+1}}{\sum_{i=1}^{m} a_{i}^{\theta+1}} .\end{cases}
\end{aligned}
$$


Let $\xi=\left(\xi_{1}, \xi_{2}, \ldots, \xi_{m}\right)$ such that $\sum_{i=1}^{m} \xi_{i}=1$ and $\xi_{i} \in(0,1)$ for $1 \leq i \leq m$ and $m \geq 2$. Then the first derivative of the function $f(x)=\sum_{i=1}^{m} \xi_{i}^{x}$ is $f^{\prime}(x)=\sum_{i=1}^{m} \xi_{i}^{x} \ln \xi_{i}<0$, which implies that the function $f(x)$ is strictly decreasing on $(-\infty, \infty)$. Since $f(1)=1$, it follows that $f(x) \lesseqgtr$ 1 if and only if $x \gtreqless 1$. This means that

$$
\sum_{i=1}^{m} \xi_{i}^{x} \lesseqgtr 1, \quad x \gtreqless 1
$$

Replacing $\xi_{i}=\frac{a_{i}}{\sum_{i=1}^{m} a_{i}}$ and $x=\theta+1$ in the above inequality yields

$$
\sum_{i=1}^{m}\left(\frac{a_{i}}{\sum_{i=1}^{m} a_{i}}\right)^{\theta+1} \lesseqgtr 1, \quad \theta \gtreqless 0 .
$$

This can be further rewritten as

$$
\sum_{i=1}^{m} a_{i}^{\theta+1} \lesseqgtr\left(\sum_{i=1}^{m} a_{i}\right)^{\theta+1}, \quad \theta \gtreqless 0, a_{i}>0, m \geq 2 .
$$

Considering inequality (3.5) reveals that

(1) when $\theta=0$, we have

$$
\lim _{x \rightarrow \infty}[\ln \mathcal{Q}(x)]^{\prime}=\varrho \sum_{i=1}^{m} a_{i} \ln p_{i}+ \begin{cases}\ln \frac{\left(\sum_{i=1}^{m} a_{i}\right)^{\sum_{i=1}^{m} a_{i}}}{\prod_{i=1}^{m} a_{i}^{a_{i}}}+0, & \rho=1 \\ \ln \frac{\left(\sum_{i=1}^{m} a_{i}\right)_{i=1}^{\sum_{i=1}^{m} a_{i}}}{\left(\prod_{i=1}^{m} a_{i}^{a_{i}}\right)^{\rho}}+\infty, & \rho<1\end{cases}
$$

(2) when $\theta>0$ and $\rho \leq 1$, we have

$$
\lim _{x \rightarrow \infty}[\ln \mathcal{Q}(x)]^{\prime}=\varrho \sum_{i=1}^{m} a_{i} \ln p_{i}+\ln \frac{\left(\sum_{i=1}^{m} a_{i}\right)^{\left(\sum_{i=1}^{m} a_{i}\right)^{\theta+1}}}{\left(\prod_{i=1}^{m} a_{i}^{a_{i}^{\theta+1}}\right)^{\rho}}+\infty=\infty
$$

Hence, when $\theta=0$ and $\rho<1$ or when $\theta>0$ and $\rho \leq 1$, we obtain

$$
\lim _{x \rightarrow \infty}\left[\ln \mathcal{Q}_{m, a, p, \rho, \varrho, \theta}(x)\right]^{\prime}=\infty
$$

when $\theta=0$ and $\rho=1$, we have

$$
\begin{aligned}
\lim _{x \rightarrow \infty}[\ln \mathcal{Q}(x)]^{\prime} & =\varrho \sum_{i=1}^{m} a_{i} \ln p_{i}+\ln \frac{\left(\sum_{i=1}^{m} a_{i}\right)^{\sum_{i=1}^{m} a_{i}}}{\prod_{i=1}^{m} a_{i}^{a_{i}}} \\
& =(\varrho-1) \sum_{i=1}^{m} a_{i} \ln p_{i}+\left(\sum_{i=1}^{m} p_{i} \frac{a_{i}}{p_{i}}\right) \ln \left(\sum_{i=1}^{m} p_{i} \frac{a_{i}}{p_{i}}\right)-\sum_{i=1}^{m} p_{i} \frac{a_{i}}{p_{i}} \ln \frac{a_{i}}{p_{i}}
\end{aligned}
$$

Let $f$ be a convex function on an interval $I \subseteq \mathbb{R}$, let $m \geq 2$ and $x_{i} \in I$ for $1 \leq i \leq m$, and let $q_{i}>0$ for $1 \leq i \leq m$. Then

$$
f\left(\frac{1}{\sum_{i=1}^{m} q_{i}} \sum_{i=1}^{m} q_{i} x_{i}\right) \leq \frac{1}{\sum_{i=1}^{m} q_{i}} \sum_{i=1}^{m} q_{i} f\left(x_{i}\right) .
$$


This inequality is called Jensen's discrete inequality for convex functions in the literature [13, Sect. 1.4] and [14, Chapter I]. Applying (3.6) to $f(x)=x \ln x$ which is convex on $(0, \infty)$, $x_{i}=\frac{a_{i}}{p_{i}}$, and $q_{i}=p_{i}$ leads to

$$
\left(\sum_{i=1}^{m} p_{i} \frac{a_{i}}{p_{i}}\right) \ln \left(\sum_{i=1}^{m} p_{i} \frac{a_{i}}{p_{i}}\right) \leq \sum_{i=1}^{m} p_{i} \frac{a_{i}}{p_{i}} \ln \frac{a_{i}}{p_{i}}
$$

Accordingly,

$$
\lim _{x \rightarrow \infty}[\ln \mathcal{Q}(x)]^{\prime} \leq(\varrho-1) \sum_{i=1}^{m} a_{i} \ln p_{i} \leq 0, \quad \varrho \geq 1 .
$$

Consequently, when $\theta=0, \rho=1$, and $\varrho \geq 1$, the function $\mathcal{Q}_{m, a, p, \rho, \varrho, \theta}(x)$ is logarithmically completely monotonic on $(0, \infty)$.

The limit

$$
\lim _{x \rightarrow 0^{+}}\left[\ln \mathcal{Q}_{m, a, p, 1,0,0}(x)\right]^{\prime}=0
$$

obtained above implies that $\left[\ln \mathcal{Q}_{m, a, p, 1,0,0}(x)\right]^{\prime} \geq 0, \mathcal{Q}_{m, a, p, 1,0,0}(x)$ is increasing, and then $\left[\ln \mathcal{Q}_{m, a, p, 1,0,0}(x)\right]^{\prime}$ is a Bernstein function on $(0, \infty)$.

When $(\rho, \varrho, \theta) \in S$, the limits

$$
\lim _{x \rightarrow 0^{+}}\left[\ln \mathcal{Q}_{m, a, p, \rho, \varrho, \theta}(x)\right]^{\prime}<0
$$

and

$$
\lim _{x \rightarrow \infty}\left[\ln \mathcal{Q}_{m, a, p, \rho, Q, \theta}(x)\right]^{\prime}=\infty
$$

derived above mean that the first derivative $\left[\ln \mathcal{Q}_{m, a, p, \rho, e, \theta}(x)\right]^{\prime}$ has a unique zero on $(0, \infty)$, that is, the functions $\ln \mathcal{Q}_{m, a, p, \rho, e, \theta}(x)$ and $\mathcal{Q}_{m, a, p, \rho, e, \theta}(x)$ have a unique minimum on $(0, \infty)$. The proof of Theorem 3.1 is complete.

\section{An open problem}

Let $m, n \geq 2, \rho, \varrho, \theta \in \mathbb{R}$, let $\lambda=\left(\lambda_{i j}\right)_{m \times n}$ with $\lambda_{i j}>0$ for $1 \leq i \leq m$ and $1 \leq j \leq n$, let $v_{i}=\sum_{j=1}^{n} \lambda_{i j}$ and $\tau_{j}=\sum_{i=1}^{m} \lambda_{i j}$ for $1 \leq i \leq m$ and $1 \leq j \leq n$, and let $p=\left(p_{i j}\right)_{m \times n}$ with $\sum_{i=1}^{m} \sum_{j=1}^{n} p_{i j}=1$ and $p_{i j} \in(0,1)$ for $1 \leq i \leq m$ and $1 \leq j \leq n$. Define

$$
Q_{m, n ; \lambda ; p ; \rho ; \varrho ; \theta}(x)=\frac{\prod_{i=1}^{m}\left[\Gamma\left(1+v_{i} x\right)\right]^{\nu_{i}^{\theta}} \prod_{j=1}^{n}\left[\Gamma\left(1+\tau_{j} x\right)\right]^{\tau_{j}^{\theta}}}{\prod_{i=1}^{m} \prod_{j=1}^{n}\left[\Gamma\left(1+\lambda_{i j} x\right)\right]^{\rho \lambda_{i j}^{\theta}}}\left(\prod_{i=1}^{m} p_{i j}^{\lambda_{i j}}\right)^{\varrho x}
$$

on the infinite interval $(0, \infty)$.

Can one find monotonicity properties for the function $Q_{m, n ; \lambda ; p ; \rho ; \rho ; \theta}(x)$ defined in equation (4.1)?

Remark 4.1 This paper is a slightly revised version of the electronic preprint [30]. 


\section{Acknowledgements}

The authors are thankful to anonymous referees for their careful corrections, helpful suggestions, and valuable comments on the original version of this paper.

\section{Funding}

The second author was partially supported by the National Research Foundation of Korea under Grant NRF-2018R1D1A1B07041846, South Korea.

\section{Availability of data and materials}

Not applicable.

\section{Competing interests}

None of the authors has any competing interests.

\section{Authors' contributions}

All authors contributed equally to the manuscript and read and approved the final manuscript.

\section{Author details}

${ }^{1}$ Institute of Mathematics, Henan Polytechnic University, Jiaozuo, China. ${ }^{2}$ College of Mathematics, Inner Mongolia University for Nationalities, Tongliao, China. ${ }^{3}$ School of Mathematical Sciences, Tianjin Polytechnic University, Tianjin, China. ${ }^{4}$ Department of Mathematics Education, Andong National University, Andong, Republic of Korea.

\section{Publisher's Note}

Springer Nature remains neutral with regard to jurisdictional claims in published maps and institutional affiliations.

Received: 19 February 2020 Accepted: 20 April 2020 Published online: 01 May 2020

\section{References}

1. Abramowitz, M., Stegun, I.A. (eds.): Handbook of Mathematical Functions with Formulas, Graphs, and Mathematical Tables, National Bureau of 10th printing edn. Standards, Applied Mathematics Series, vol. 55. Dover, New York (1972)

2. Alzer, H.: Complete monotonicity of a function related to the binomial probability. J. Math. Anal. Appl. 459, 10-15 (2018). https://doi.org/10.1016/j.jmaa.2017.10.077

3. Atanassov, R.D., Tsoukrovski, U.V.: Some properties of a class of logarithmically completely monotonic functions. C. R. Acad. Bulgare Sci. 41, 21-23 (1988)

4. Berg, C.: Integral representation of some functions related to the gamma function. Mediterr. J. Math. 1, 433-439 (2004). https://doi.org/10.1007/s00009-004-0022-6

5. Chen, Y.-C., Mansour, T., Zou, Q.: On the complete monotonicity of quotient of gamma functions. Math. Inequal. Appl. 15, 395-402 (2012). https://doi.org/10.7153/mia-15-33

6. Chung, W.S., Kim, T., Mansour, T.: The $q$-deformed gamma function and $q$-deformed polygamma function. Bull. Korean Math. Soc. 51, 1155-1161 (2014). https://doi.org/10.4134/BKMS.2014.51.4.1155

7. Guo, B.-N., Qi, F.: A property of logarithmically absolutely monotonic functions and the logarithmically complete monotonicity of a power-exponential function. Sci. Bull. "Politeh." Univ. Buchar., Ser. A, Appl. Math. Phys. 72, 21-30 (2010)

8. Guo, B.-N., Qi, F.: Two new proofs of the complete monotonicity of a function involving the psi function. Bull. Korean Math. Soc. 47, 103-111 (2010). https://doi.org/10.4134/bkms.2010.47.1.103

9. Guo, B.-N., Qi, F., Zhao, J.-L., Luo, Q.-M.: Sharp inequalities for polygamma functions. Math. Slovaca 65, 103-120 (2015). https://doi.org/10.1515/ms-2015-0010

10. Krasniqi, V., Mansour, T., Shabani, A.Sh.: Some monotonicity properties and inequalities for $\Gamma$ and $\zeta$-functions. Math. Commun. 15, 365-376 (2010)

11. Leblanc, A., Johnson, B.C.: On a uniformly integrable family of polynomials defined on the unit interval. J. Inequal. Pure Appl. Math. 8(3), Article ID 67 (2007) https://www.emis.de/journals/JIPAM/article878.html

12. Leblanc, A., Johnson, B.C.: A family of inequalities related to binomial probabilities. Tech. Report, Department of Statistics, University of Manitoba, 2006-03

13. Mitrinović, D.S.: Analytic Inequalities. Die Grundlehren der mathematischen Wissenschaften, vol. 165. Springer, New York (1970) In cooperation with P.M. Vasić

14. Mitrinović, D.S., Pečarić, J.E., Fink, A.M.: Classical and New Inequalities in Analysis. Kluwer Academic, Norwell (1993). https://doi.org/10.1007/978-94-017-1043-5

15. Olver, F.W.J., Lozier, D.W., Boisvert, R.F., Clark, C.W. (eds.): NIST Handbook of Mathematical Functions Cambridge University Press, New York (2010). Available online at http://dlmf.nist.gov/

16. Ouimet, F.: Complete monotonicity of multinomial probabilities and its application to Bernstein estimators on the simplex. J. Math. Anal. Appl. 466, 1609-1617 (2018). https://doi.org/10.1016/j.jmaa.2018.06.049

17. Ouimet, F:: Complete monotonicity of a ratio of gamma functions and some combinatorial inequalities for multinomial coefficients (2019). arXiv preprint, available online at https://arxiv.org/abs/1907.05262

18. Qi, F.: Limit formulas for ratios between derivatives of the gamma and digamma functions at their singularities. Filomat 27,601-604 (2013). https://doi.org/10.2298/FIL1304601Q

19. Qi, F.: A logarithmically completely monotonic function involving the q-gamma function (2018). HAL preprint, available online at https://hal.archives-ouvertes.fr/hal-01803352v1

20. Qi, F.: A double inequality for the ratio of two non-zero neighbouring Bernoulli numbers. J. Comput. Appl. Math. 351, 1-5 (2019). https://doi.org/10.1016/j.cam.2018.10.049

21. Qi, F.: Complete monotonicity for a new ratio of finite many gamma functions (2020). HAL preprint, available online at https://hal.archives-ouvertes.fr/hal-02511909v1 
22. Qi, F., Agarwal, R.P.: On complete monotonicity for several classes of functions related to ratios of gamma functions. J. Inequal. Appl. 2019, Paper No. 36, 42 pages (2019) https://doi.org/10.1186/s13660-019-1976-z

23. Qi, F., Chapman, R.J.: Two closed forms for the Bernoulli polynomials. J. Number Theory 159, 89-100 (2016). https://doi.org/10.1016/j.jnt.2015.07.021

24. Qi, F., Chen, C.-P.: A complete monotonicity property of the gamma function. J. Math. Anal. Appl. 296, 603-607 (2004) https://doi.org/10.1016/i.jmaa.2004.04.026

25. Qi, F., Guo, B.-N.: Complete monotonicity of divided differences of the di- and tri-gamma functions with applications. Georgian Math. J. 23, 279-291 (2016). https://doi.org/10.1515/gmj-2016-0004

26. Qi, F., Guo, B.-N.: Integral representations and complete monotonicity of remainders of the Binet and Stirling formulas for the gamma function. Rev. R. Acad. Cienc. Exactas Fís. Nat., Ser. A Mat. 111, 425-434 (2017). https://doi.org/10.1007/s13398-016-0302-6

27. Qi, F., Guo, B.-N.: From inequalities involving exponential functions and sums to logarithmically complete monotonicity of ratios of gamma functions (2020). arXiv preprint, available online at https://arxiv.org/abs/2001.02175v1

28. Qi, F., Li, W.-H.: Integral representations and properties of some functions involving the logarithmic function. Filomat 30, 1659-1674 (2016). https://doi.org/10.2298/FIL1607659Q

29. Qi, F., Li, W.-H., Yu, S.-B., Du, X.-Y., Guo, B.-N.: A ratio of many gamma functions and its properties with applications (2019). arXiv preprint, available online at https://arXiv.org/abs/1911.05883v1

30. Qi, F., Lim, D.: Monotonicity properties for a ratio of finite many gamma functions (2020). HAL preprint, available online at https://hal.archives-ouvertes.fr/hal-02511883v1

31. Qi, F., Liu, A.-Q:: Completely monotonic degrees for a difference between the logarithmic and psi functions. J. Comput. Appl. Math. 361, 366-371 (2019). https://doi.org/10.1016/j.cam.2019.05.001

32. Qi, F., Niu, D.-W., Lim, D., Guo, B.-N.: Some logarithmically completely monotonic functions and inequalities for multinomial coefficients and multivariate beta functions (2018). HAL preprint, available online at https://hal.archives-ouvertes.fr/hal-01769288v1

33. Schilling, R.L., Song, R., Vondraček, Z:: Bernstein Functions-Theory and Applications, 2nd edn. de Gruyter Studies in Mathematics, vol. 37. de Gruyter, Berlin (2012). https://doi.org/10.1515/9783110269338.

34. Temme, N.M.: Special Functions: An Introduction to Classical Functions of Mathematical Physics. Wiley-Interscience, New York (1996). https://doi.org/10.1002/9781118032572

35. Widder, D.V.: The Laplace Transform. Princeton University Press, Princeton (1946)

36. Yang, Z.-H., Tian, J.-F.: A class of completely mixed monotonic functions involving the gamma function with applications. Proc. Am. Math. Soc. 146, 4707-4721 (2018). https://doi.org/10.1090/proc/14199

\section{Submit your manuscript to a SpringerOpen ${ }^{\circ}$ journal and benefit from:}

- Convenient online submission

- Rigorous peer review

- Open access: articles freely available online

- High visibility within the field

- Retaining the copyright to your article

Submit your next manuscript at $\gg$ springeropen.com 